\title{
The comparative study of analyzing the T-shaped and square-shaped concrete columns
}

\author{
Ade Sri Wahyuni ${ }^{1 *}$, Mukhlis Islam $^{1}$, and Rizky Arian Putra ${ }^{1}$ \\ ${ }^{1}$ Civil Engineering Department, University of Bengkulu, Indonesia
}

\begin{abstract}
This study aims to analyze how much changes in column shape effect have on the strength of the column structure. This calculation is comparing a square-shaped concrete column with two different axis of a T-shaped column. Each sample is compared in terms of 6 different column dimensions, however, the cross-sectional area within the three samples ( Square, T-shaped axis1 and 2) has remained constant. The concrete quality (fc') is kept at $30 \mathrm{MPa}$ while the reinforcement area used $1 \%$ and $5 \%$. Column calculation analysis is using Hognestad stress-strain diagram relationship and finite difference method. The result shows, the greater the reinforcement ratio, the greater the difference of $\mathrm{Mn}$ value and the smaller the difference of Pn value, of T-shaped and square column for each area. The same cross-sectional area does not necessarily lead to the same behavior of a Tshaped column with a different axis
\end{abstract}

\section{Introduction}

Structural design plays an important role to ensure the strength, safety, and durability of the building. The column is one of the structural components of a building whose main task is to support axial loads $(\mathrm{Pn})$ and nominal moments $(\mathrm{Mn})$. Column design is critical in structural design as the column failure may result in the collapse of other structural components associated with it [1].

The cross-sectional dimension of the column is small relative to its overall length. Structural concrete columns are used in buildings to support the beam and floor system and transfer the vertical live loads and dead loads from the upper structure into the footings and foundations.[2]

Symmetrical reinforcement is generally used in column planning to avoid mistakes during the installation of reinforcement. This symmetrical reinforcement is also needed if there is a possibility of alternating forces on the structure, such as earthquake loads

A T-shaped column is used in the building as an alternative for maximizing room function and aesthetic issues. Using a T-shaped column will create more space in the room without the appearance of the big column. Another use of a T-shaped column is in the goods elevator with alternating doors. In this case, the T-shaped column cannot be replaced by the shear wall

\section{Literature Review}

Columns usually carry bending moments about both principal axis and so it is common to place reinforcing bars in all four faces. The bars in the side faces of this column of course contribute to the strength of the column [2].

Fig. 1 shows the Stress-strain diagram of the square column. The ratio of longitudinal reinforcement in a column with a rectangular cross-section using the Hognestad stress-strain relationship

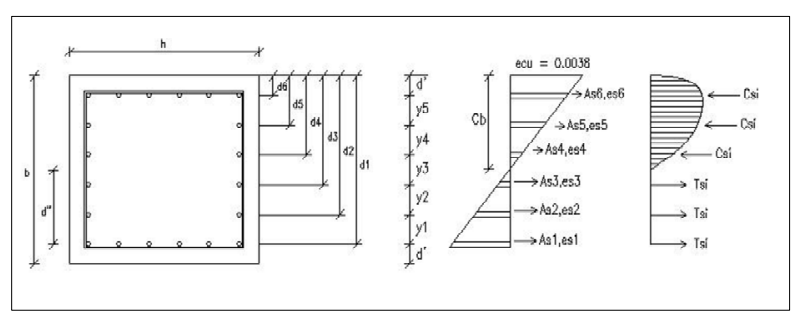

Fig. 1. Square column

When a section has bars distributed at all faces, the derivation of equations for design and analysis becomes difficult because the bars may be at various stress levels throughout the section. The analysis of such a section can be carried out using the requirements of strain compatibility and equilibrium.[3]

To take account of the bars in the side faces we simply have to consider additional layers of steel at various levels. Steel areas $\mathrm{As}_{1}, \mathrm{As}_{2}, \mathrm{As}_{3}, \mathrm{As}_{4}, \mathrm{As}_{5}, \mathrm{As}_{6}$ are located at depth $d_{1}, d_{2}, d_{3}, d_{4}, d_{5}, d_{6}$. The steel layers will be in tension or compression and maybe at yield or elastic, depending on the distance from the neutral axis. As the neutral axis may be placed in various positions in the section, some steel layers may be in either compression or tension [3].

\footnotetext{
* Corresponding Author: ade.sri.w@unib.ac.id
} 
For a general bar I in the section, the strain diagram indicates that

$$
\varepsilon_{s}=0,003 \frac{c-d i}{c}
$$

Where compressive strains are positive, and tensile strains are negative. Then the stress $\mathrm{f}_{\mathrm{si}}$ in bar $\mathrm{i}$ is given by the following relationship [2][3][4].

$$
\begin{aligned}
& \text { If } \quad \varepsilon_{s i} \geq \frac{f y}{E s} \quad f_{s i}=f_{y} \\
& \text { Or } \quad \text { if } \frac{f y}{E s}>\varepsilon_{s i}>-\frac{f y}{E s} \quad f_{s}=\varepsilon_{s} . E_{s} \\
& \text { If } \varepsilon_{s i} \leq-\frac{f y}{E s} \quad \quad f_{s i}=f_{y} \\
& C_{s}=A_{s}^{\prime} \cdot f_{s}^{\prime} \\
& T_{s}=A_{s} f_{s} \\
& \text { (2.1) } \\
& C_{c}=0,85 \cdot f^{\prime} \text { c.a.b } \\
& P n=C_{c}+C_{s}-T_{s} \\
& M n=P n . e
\end{aligned}
$$

\section{Research aim}

This study aims to produce the interaction diagram PnMn of the square column compared with T shaped column with two different axis

Fig. 2 shows the T-shaped Column in the first axis and will be named T1 while the second axis is shown in Fig. 3 and named T2

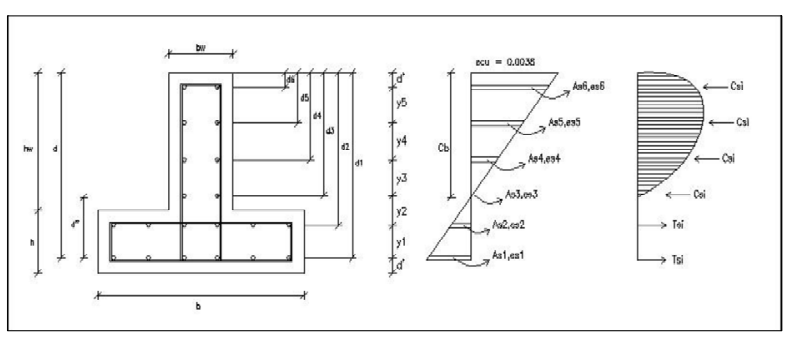

Fig. 2. T-shaped column first axis

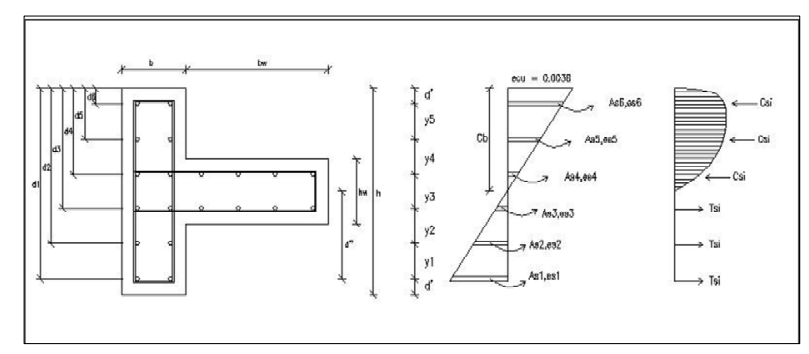

Fig. 3 T-shaped column second axis

Since there are several layers of steel, there will not be a single sharp discontinuity in the interaction diagram at the balanced failure point, rather a more curved diagram will result because not all the tension reaches the yield strength at the same time [2,3]
The foregoing approach for calculating interaction diagrams by determining the combinations for Pn and Mn at failure for various neutral axis locations can also be used for columns of shapes other than rectangular and walls, However when the neutral axis depth is small, such as in flanged walls, and the dimensions of the crosssection are large, very large tensile strains can occur in the far layers of the tension steel $[2,3]$

If the maximum strength of the section is to be calculated, it is important to determine whether the tensile strains in these bars is such that the bars have entered the strain-hardening range, If the full stress-strain curve for the steel is known, the actual stresses corresponding to the strain levels can be used in the strength calculations, The additional flexural strength due to strain hardening should be taken into account when the resulting over strength could lead to an alternative brittle failure (e.g., a shear failure rather than a flexural failure. For nonsymmetrical sections or steel arrangement, two interaction curves will result, one for each sense of the eccentricity $[2,3]$

\section{Limitation}

The limitation available in this study are:

Reinforced concrete with a square cross-section with four-face longitudinal reinforcement.

The reinforced concrete column that only experiences a combination of uniaxial bending moments

Concrete quality fc' $30 \mathrm{MPa}$

Reinforcement area $1 \%$ and $5 \%$ of the column crosssectional area

The reinforcement quality fy $400 \mathrm{MPa}$.

$\mathrm{E}_{\mathrm{s}}=200.000 \mathrm{MPa}$

\section{Methods}

The flange dimension of $\mathrm{T}$ shaped column is $25 \times 80,25 \mathrm{x}$ $90,30 \times 80,30 \times 90,35 \times 80,35 \times 90$, and the cross-sectional area of these three columns are kept constant.

The interaction diagram of Pn-Mn will be compared with two different reinforcement areas $1 \%$ and $5 \%$. This data analysis uses some basic assumptions commonly used in the calculation of column structure.

The iteration method is used repeatedly to get a graph at certain points. The distance between the neutral line and the edge of the compressed concrete fiber $\mathrm{c}$ is assumed to be 20 pieces for one value of $\mathrm{c}$ divided into 100 segments. The calculation will stop when it has reached 100 segments with one value of $\mathrm{c}$ producing one axial force and nominal moment.

The points of each value of $\mathrm{C}$ are combined to form the interaction diagram of the axial force $(\mathrm{Pn})$ and nominal moment (Mn).

\section{Results and Discussion}

Any value of $d_{n}$ less than $d$ will produce a valid point on the strength line and a pair of values $\mathrm{Pn}$ - Mn which represent a condition of failure of the section. If very small values are chosen for $d_{n}$ i.e less than the value at which 
pure bending failure occurs, a negative value for Pn will be obtained. This represents failure under a combination of moment and axial tension by the strength line extending down into the fourth quadrant $[2,3]$.

The strength line depends on the dimensions, reinforcement details, and material properties for the column cross-section and is a section property, irrespective of whether the column is short or long[2, 3].

The small compressive force increases the moment carrying capacity of the section so that the interaction diagram 'bulges' outwards at intermediate values of Pn. In such cases, the axial force Pn is reducing the tensile force in the steel, which is the primary cause of failure $[2,3]$

The balance failure condition lies close to the extremity of the bulge of the interaction diagram so that the balance moment is approximately the maximum moment that the section can carry in the presence of axial compression[2, 3]

Fig.4 until Fig. 5 shows the interaction diagram of the Square column, T-shaped column first axis, and Tshaped column second axis. The square column becomes the benchmark of the axial force and moment that are allowable and can be supported by the column

The points within the inside of the red curve are also pairs of axial loads and moments that can be supported, but these pairs of loads are lower than the bearing capacity of the column, so if used, it means that the dimensions \& reinforcement of the column are overdesigned.

The point outside the red curve, are pairs of axial loads and moments that exceed the bearing capacity of the column so that the column is not allowed to support these loads. Fig.4 and Fig.5 show interaction diagrams between columns with the same cross-sectional area, with the flange size of T column $250 \times 800$.

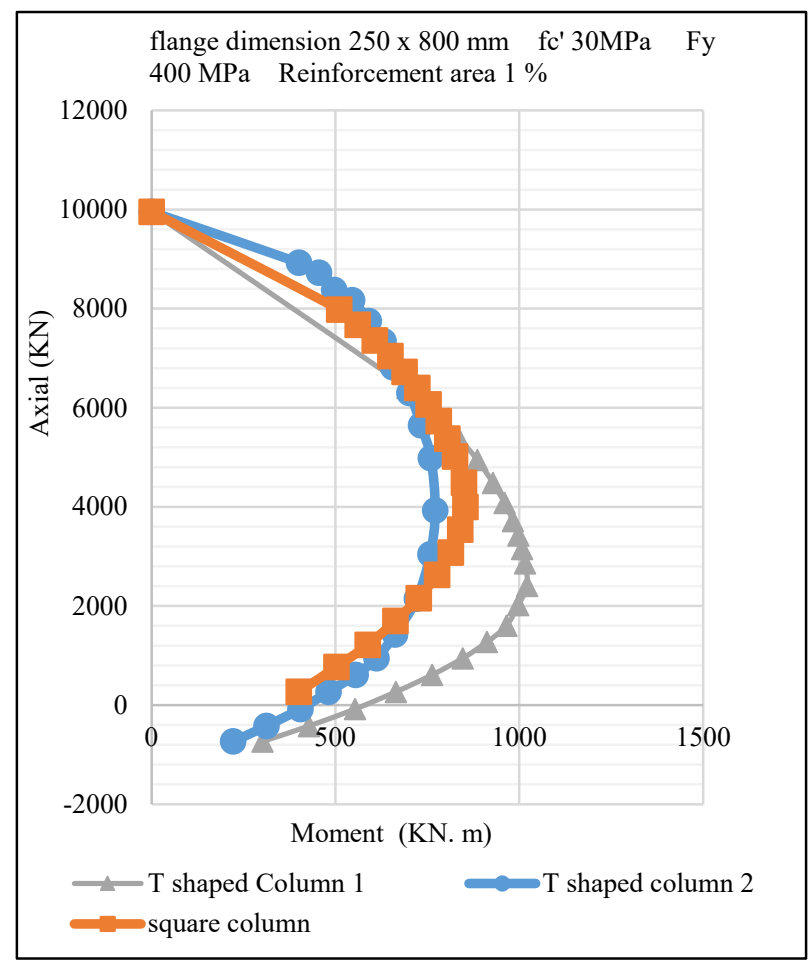

Fig. 4. Interaction diagram $A$

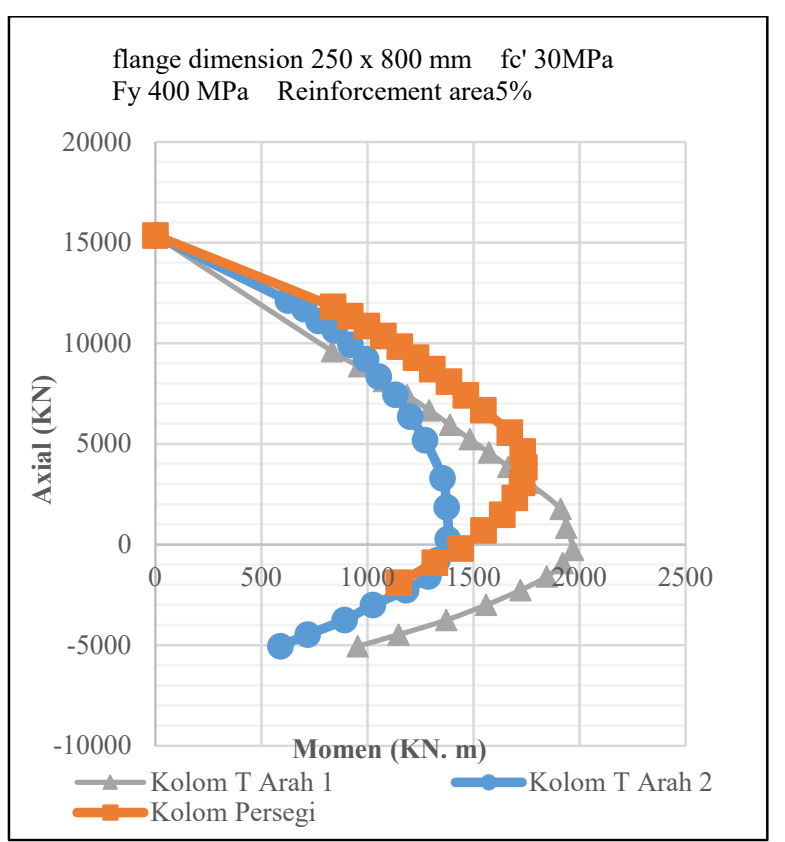

Fig. 5. Interaction diagram B

The difference between these two images is in the percentage of reinforcement area used. Fig. 1 uses $1 \%$ reinforcement area and Fig. 2 uses 5\% reinforcement area

Column T2 shows that about $35 \%$ of the Pn and Mn combinations are in the safe region, while column T2 shows that only $15 \%$ of the Pn and Mn combinations are in the safe region.

When reinforcement area is increased to $5 \%$ more combinations of $\mathrm{Pn}-\mathrm{Mn}$ are in a safe area. The T2column increased to approximately $70 \%$ while the T1column also increased from $10 \%$ to $45 \%$.

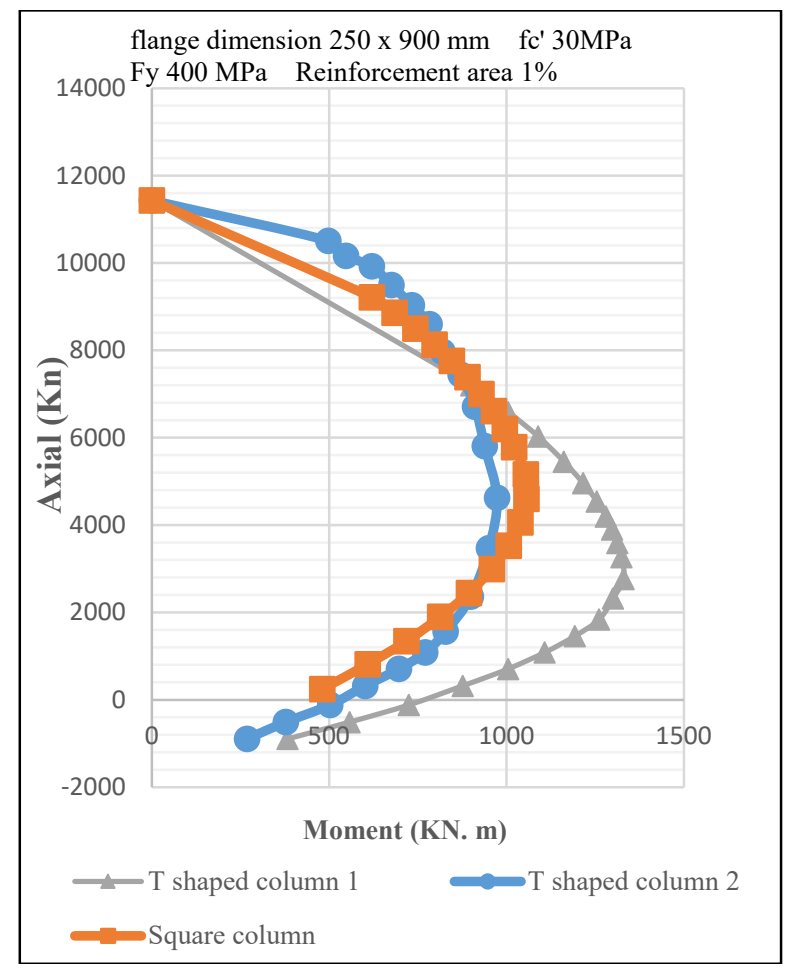

Fig. 6. Interaction diagram $\mathrm{C}$ 


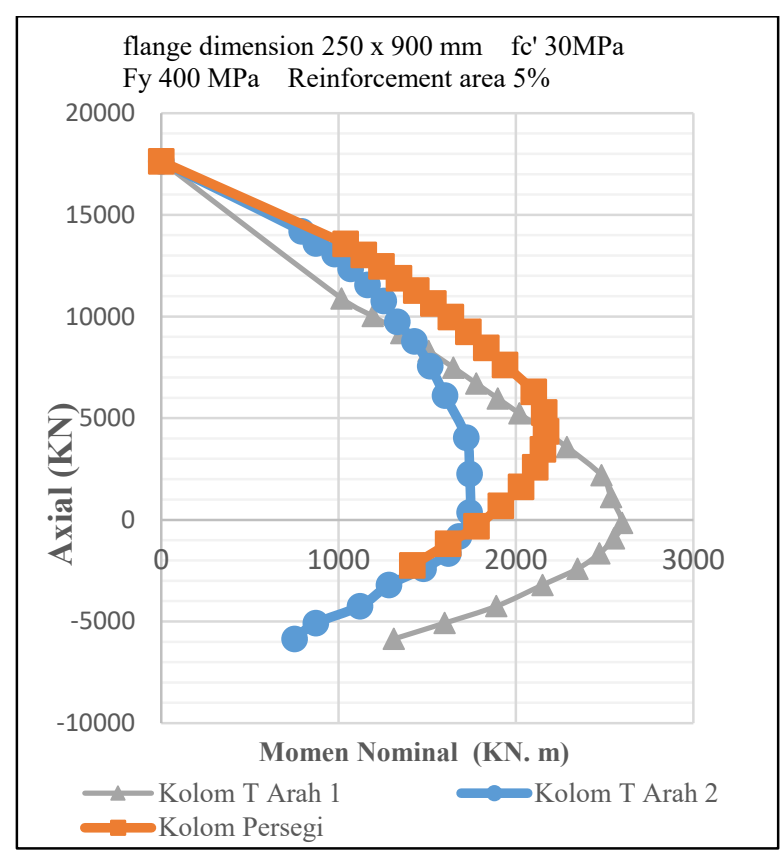

Fig. 7. Interaction diagram D

Fig. 6 and Fig. 7 show interaction diagrams between columns with the same cross-sectional area, in which the flange size of the T column is $250 \times 900$

Column T2 still shows that about $35 \%$ of the Pn and Mn combinations are in the safe region, while the safe region in column $\mathrm{T} 1$ decreases to $5 \%$ of the $\mathrm{Pn}$ and $\mathrm{Mn}$ combinations.

When reinforcement area is increased to $5 \%$ The T2-column is still approximately $70 \%$ while the T1column is still 45\%. Even though the Axial Force (Pn) and Moment increase with the bigger cross-sectional area, the percentage of the pair number of Pn-Mn which is still considered safe to hold the load is the same as the interaction diagram in Fig. 5

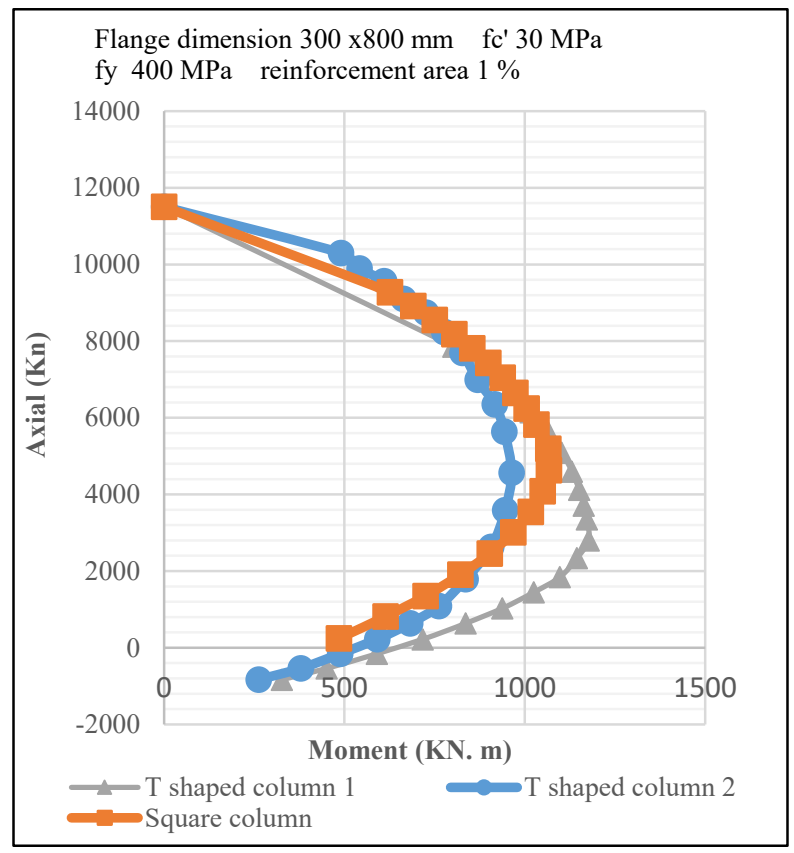

Fig. 8. Interaction diagram $\mathrm{E}$

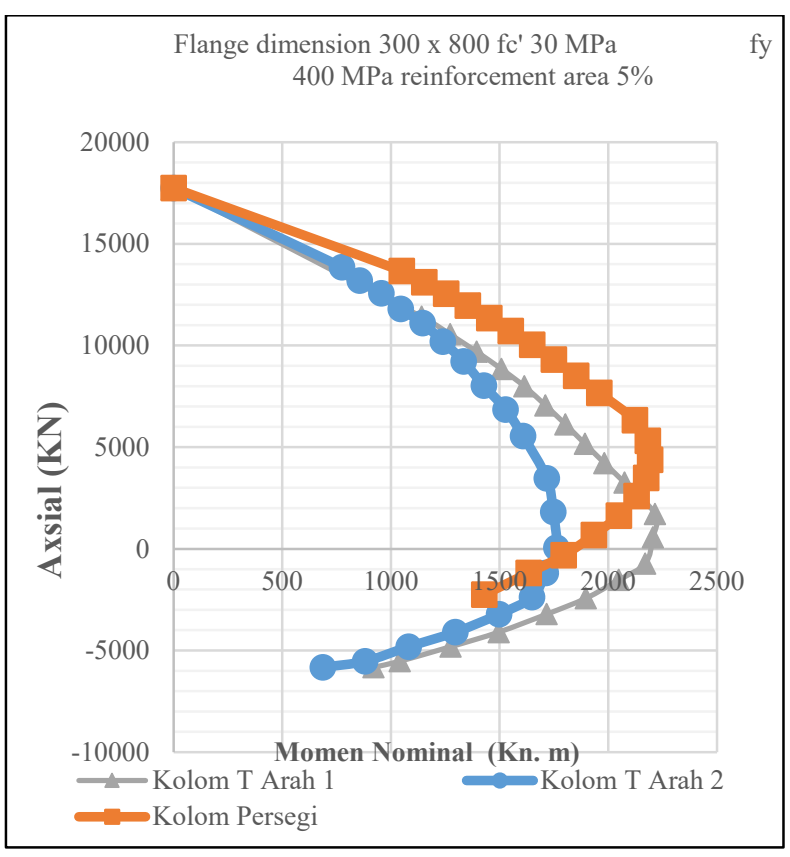

Fig. 9. Interaction diagram $F$

Fig. 8 and Fig. 9 show interaction diagrams between columns with the flange dimension of T column $300 \times 800$. Fig. 8 uses $1 \%$ reinforcement area and Fig. 9 uses 5\% reinforcement area

Column T2 shows that about $50 \%$ of the Pn and Mn combinations are exceeding the bearing capacity, while column T2 shows that only $80 \%$ of the Pn and Mn combinations are exceeding the bearing capacity.

When the reinforcement area is increased to $5 \%$ more combinations of $\mathrm{Pn} \mathrm{Mn}$ are in a safe area. The T2-column increased to approximately $60 \%$ while the T1-column also increased to $50 \%$.

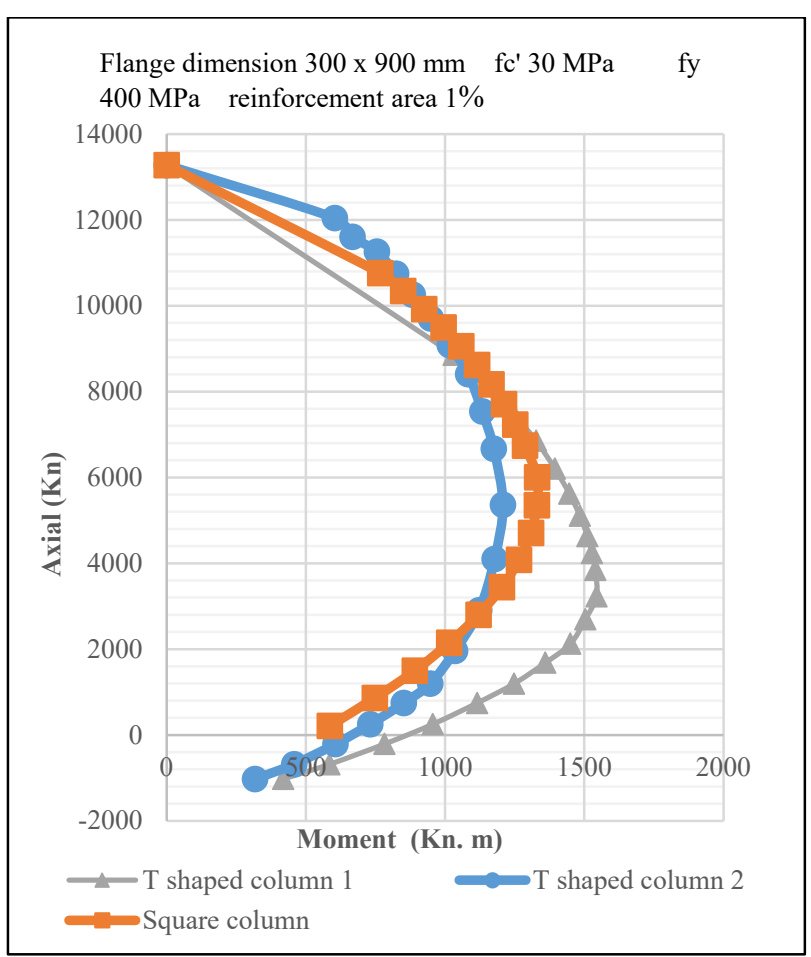

Fig. 10. Interaction diagram $G$ 


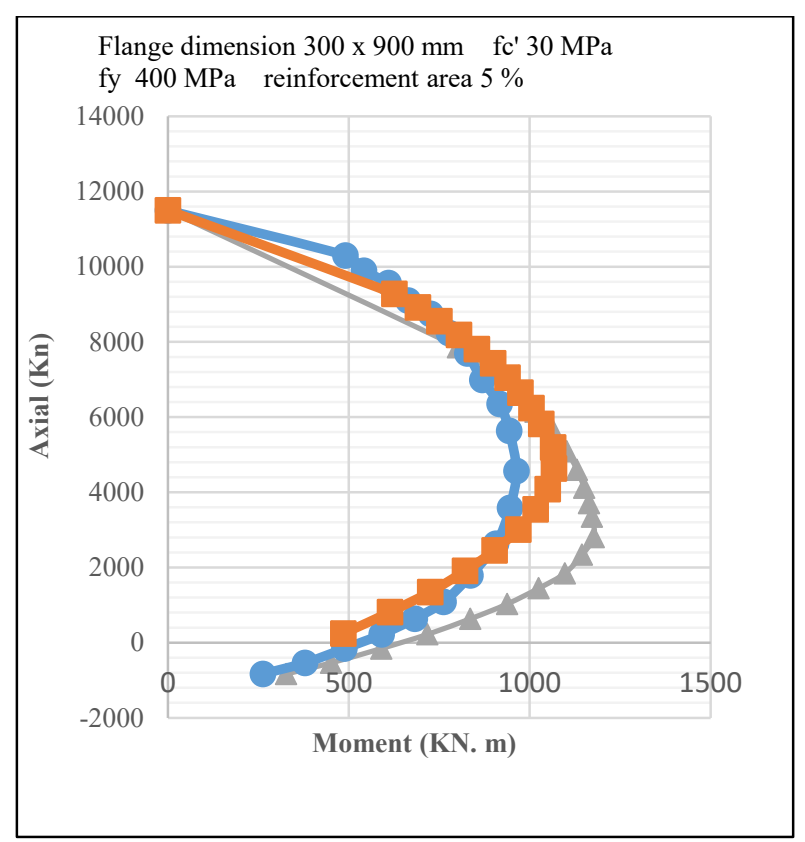

Fig.11. Interaction diagram $\mathrm{H}$

Fig. 10 and Fig. 11 show interaction diagrams between columns with the size of the flange of T column $300 \mathrm{x}$ 900

Column T2 shows that about $45 \%$ of the Pn and Mn combinations are in the safe region, while the safe region in column T2 slightly increases to $25 \%$ of the Pn and Mn combinations are in the safe region.

When reinforcement area is increased to 5\%

The T2-column is still approximately $70 \%$ while the T1-column is still $50 \%$.

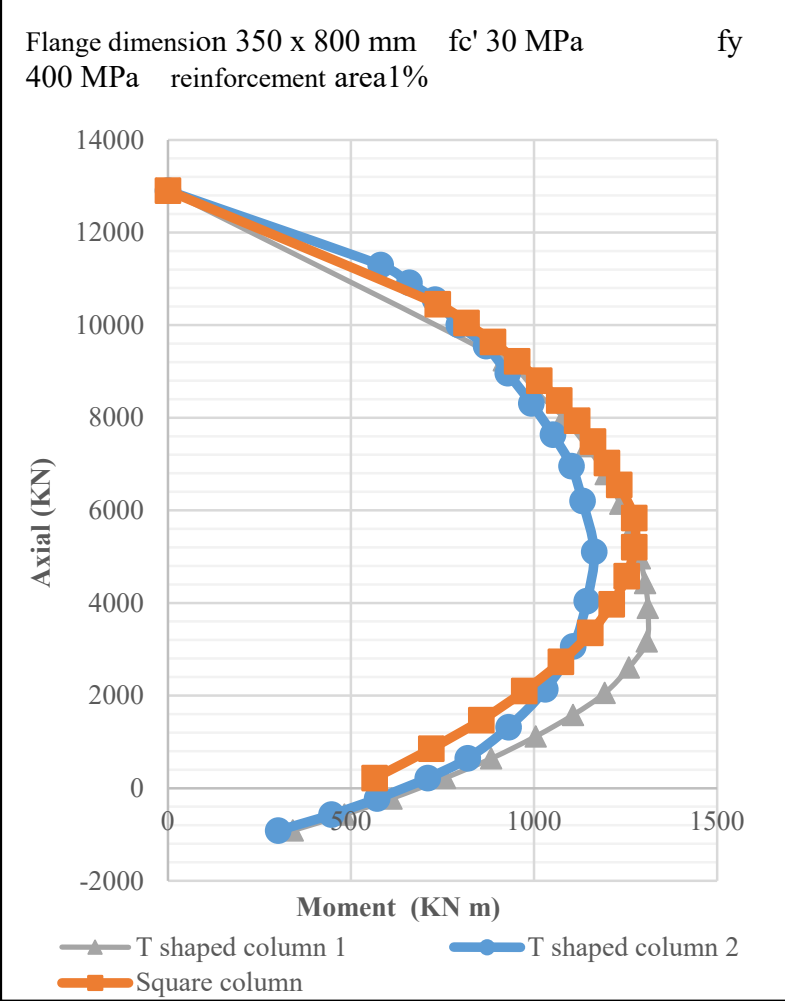

Fig. 12. Interaction diagram I

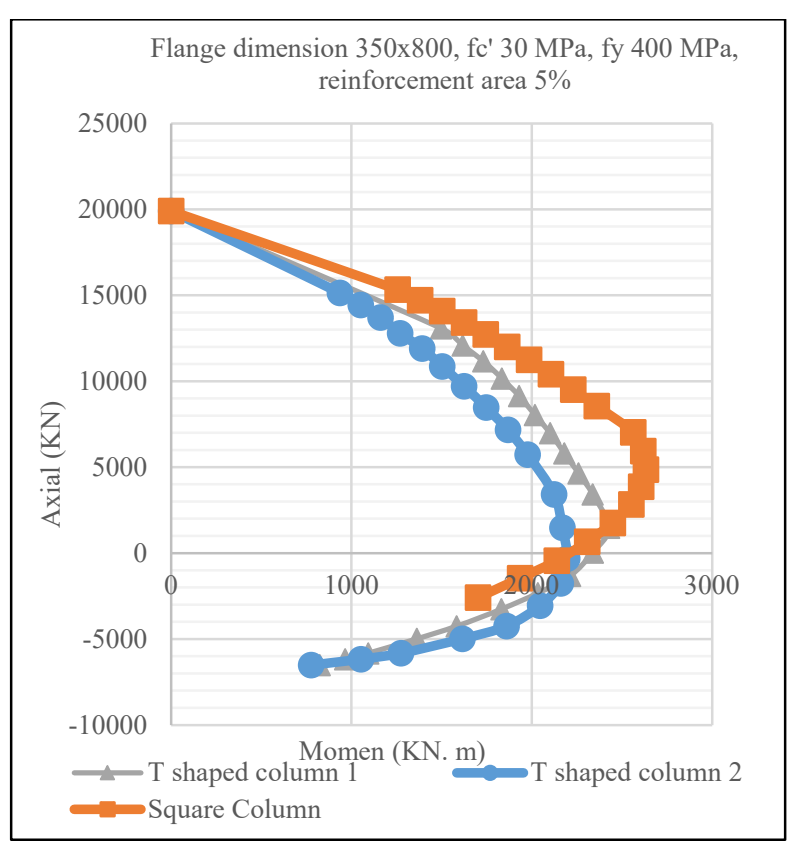

Fig. 13. Interaction diagram $\mathrm{J}$

Fig. 12 and Fig. 13 show interaction diagrams between columns, with the flange size of T column $350 \times 800$. Fig. 12 uses 1\% reinforcement area and Fig. 13 uses 5\% reinforcement area

Column T2 shows that about 55\% of the Pn and Mn combinations are in the safe region, while column T2 shows that only $35 \%$ of the Pn and Mn combinations are in the safe region.

When the reinforcement area is increased to $5 \%$ more combinations of PN Mn are in a safe area. The T2-column increased to approximately $60 \%$ while the T1-column also increased to $50 \%$.

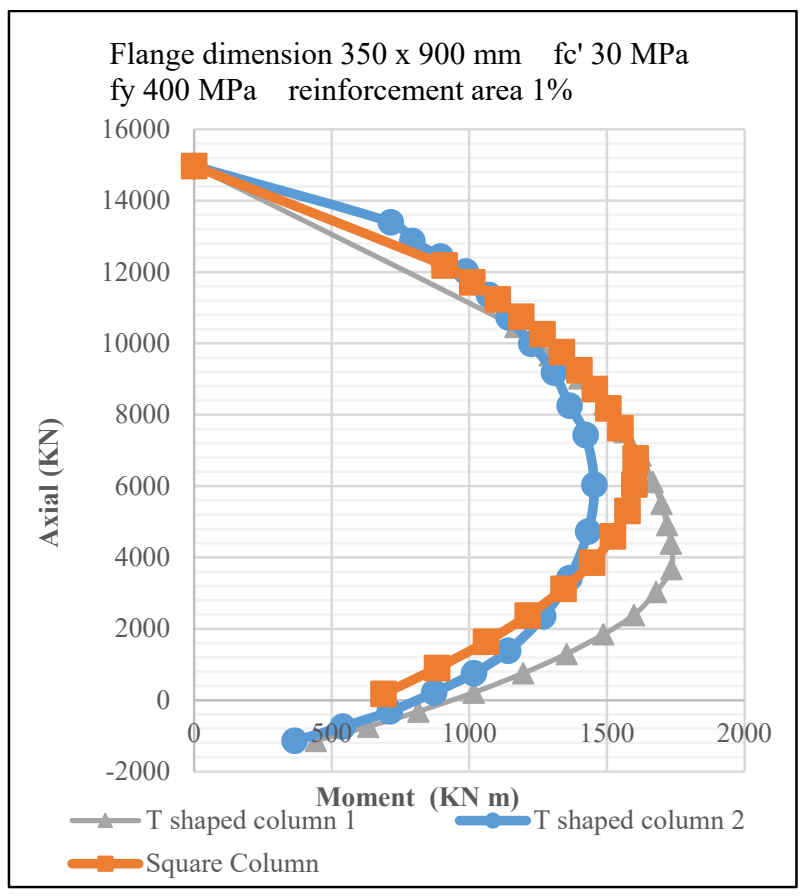

Fig. 14. Interaction diagram $K$ 


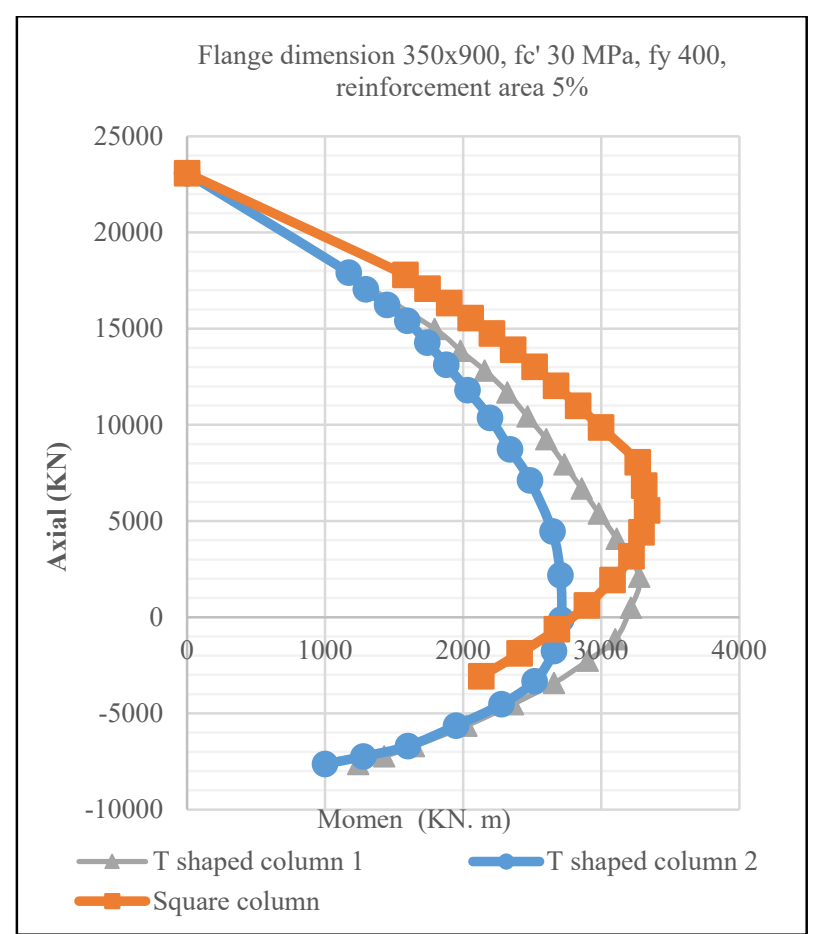

Fig.15. interaction diagram $\mathrm{L}$

Fig. 14 and Fig. 15 show interaction diagrams between columns with the flange size of T column 350x900

Column T2 shows that about $50 \%$ of the Pn and Mn combinations are in the safe region, while the safe region in column T2 slightly decreases to $25 \%$ of the Pn and Mn combinations are in the safe region.

When reinforcement area is increased to $5 \%$ The T2column is still approximately $65 \%$ while the T1column is still $50 \%$.

\section{Conclusion}

The shape of the Mn-Pn column interaction diagram is affected by an increasing amount of tensile steel.

The increase in the value of the cross-sectional area does not affect the percentage of the area that is in a safe area with a $5 \%$ reinforcement area. T1 axis ranges from 45 to $50 \%$ while the T2 axis percentage of safe areas is in the range of 60 to $70 \%$.

As for reinforcement area, $1 \%$ for T2 ranges from 35 $55 \%$, while $\mathrm{T} 1$ ranges from $5-50 \%$.

Column $\mathrm{T}$ with the most optimum area is on the flange dimensions $350 \times 800$ with the most pair of Pn-Mn which is not exceeding the bearing capacity.

The greater the reinforcement area, the bigger the cross-sectional area, the bigger the axial force and moment, T1 shows the bigger moment that exceeding the bearing capacity. However, within the same dimension but bigger reinforcement area, the T1 shows some points which allowed to hold the load.

The same cross-sectional area does not necessarily lead to the same behavior of $\mathrm{T}$ shaped column.

\section{References}

1. E. G. Nawy, Reinforced Concrete A Basic Approach, Bandung (1998)

2. R. F. Warner, B. V. Rangan, A. S, Hall, K. A. Faulkes, Concrete Structures, Longman (1999)

3. R. Park and T. Paulay, Reinforced Concrete Structures, A Wiley-Interscience Publication, (1933)

4. National Standardization Agency, SNI 28472019 Structural Concrete Requirements for Buildings, BSN (2019) 